Highlight

\title{
Recent progress on photocatalysts with wide visible light range absorption for heterogeneous water splitting
}

\author{
Shanshan Chen, Fuxiang Zhang*
}

Solar-driven water splitting for hydrogen production is an ideal way to solve the problems of the energy crisis and environment pollution, and is considered as the "Holy Grail" of chemistry [1]. Typically, photocatalytic water splitting can be divided into three processes: (1) photoabsorption and photoexcitation of the semiconductor, (2) the excited carriers separate and migrate to the surface of photocatalyst, and (3) reactions of the carriers with water to produce $\mathrm{H}_{2}$ and $\mathrm{O}_{2}$. From the viewpoint of commercial application, a promising photocatalyst should satisfy both the requirements of a correct band structure (which can drive the water splitting half reactions), and harvest light in a wide visible light range and possess good photo-stability. To increase visible light utilization, various strategies have been developed, which can be generally described as decreasing the conduction band energy, increasing the valence band energy, or mediating the positions of conduction band and valence band simultaneously. The types of the reported visible light responsive photocatalysts can be classified as cation-doped photocatalysts, anion-doped photocatalysts, and solid solution photocatalysts, etc [2].

Metal ion doping is an effective strategy to extend the absorption of most metal oxide semiconductors into the visible light region, but single element doping generally creates defect sites, which act as charge recombination centers due to the charge imbalance. To overcome this, the introduction of a second doping element is becoming more popular to broaden the light absorption range and improve the photocatalytic activity, by using its inhibition of defect sites by charge balance. Kudo et al. [3] reported that $\mathrm{SrTiO}_{3}$ co-doped with rhodium and antimony can drive overall water splitting under visible light, while $\mathrm{SrTiO}_{3}$ doped with only rhodium or antimony did not work. The main reason was the inhibition of $\mathrm{Rh}^{4+}$ ions by $\mathrm{Sb}$ co-doping. Liu et al. [4] reported a red anatase $\mathrm{TiO}_{2}$ microsphere by introducing B to weaken nearby Ti-O bonds for the easy substitution of oxygen by nitrogen. Detailed characterization indicated that $\mathrm{Ti}^{3+}$ species as recombination centers were efficiently suppressed in the red anatase $\mathrm{TiO}_{2}$ by the charge compensation effect of B. Anion doping to some layered or tunneled oxides has been also employed to extend the visible light absorption [5]. Recently, Chen et al. [6] examined the influence of co-catalysts on nitrogen-doped layered Ta-based oxides, and achieved obvious $\mathrm{H}_{2}$ or $\mathrm{O}_{2}$ evolution from the water splitting half reactions under visible light irradiation, demonstrating their potential in photocatalytic overall water splitting (Fig. 1).

Some (oxy)nitrides and oxysulfides containing a $d^{0}$ or $d^{10}$ electronic configuration have been also developed as promising wide range visible light responsive materials for water splitting by Domen et al. [7,8]. To date it has achieved overall water splitting on the single $\left(\mathrm{Ga}_{1-x} \mathrm{Zn}_{x}\right)\left(\mathrm{N}_{1-x} \mathrm{O}_{x}\right)$ solid solution, with an apparent quantum efficiency of 5.9\% at 420-440 $\mathrm{nm}$ [9] and photo-stability of 3 months ( $2160 \mathrm{~h}$ ) under visible light irradiation [10]. In addition, overall water splitting was also successful by two-step photoexcitation (so-called "Z-scheme"), and the art-of-the-state apparent quantum yield is $6.3 \%$ at $420.5 \mathrm{~nm}$ monochromatic light using $\mathrm{Pt} / \mathrm{ZrO}_{2} / \mathrm{TaON}, \mathrm{PtO}_{x} / \mathrm{WO}_{3}$, and $\mathrm{IO}_{3}^{-} / \mathrm{I}^{-}$pairs as the $\mathrm{H}_{2}$ evolution photocatalyst, $\mathrm{O}_{2}$ evolution photocatalyst and redox mediator, respectively [11]. To promote the Z-scheme conversion efficiency, continuous efforts should be made to improve the performance of the $\mathrm{H}_{2}$-evolving photocatalyst because the current quantum yield of $\mathrm{PtO}_{\mathrm{x}} / \mathrm{WO}_{3}$ for water oxidation is relatively high, reaching $20 \%$ at $420 \mathrm{~nm}$ with $\mathrm{IO}_{3}{ }^{-}$ions as an electron acceptor [12]. It is worth noting

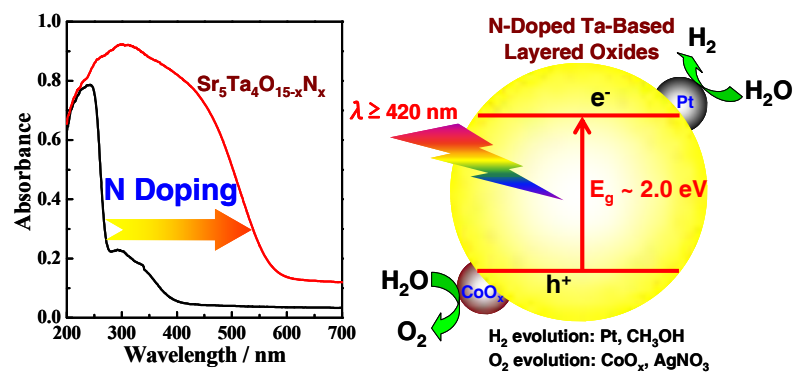

Fig. 1. UV-Vis diffuse reflectance spectra of $\mathrm{Sr}_{5} \mathrm{Ta}_{4} \mathrm{O}_{15}$ and $\mathrm{Sr}_{5} \mathrm{Ta}_{4} \mathrm{O}_{15-x} \mathrm{~N}_{x}$ samples (left), and the schematic diagram of the photocatalytic water splitting half reactions on nitrogen-doped Ta-based layered oxides. Reproduced from Ref. [6] with permission from The Royal Society of Chemistry. 
that the Z-scheme overall water splitting system without a redox mediator (( $\left.\left.\mathrm{Ru} / \mathrm{SrTiO}_{3}: \mathrm{Rh}\right)-\left(\mathrm{BiVO}_{4}\right)\right)$ has been proven feasible [13]. This should be another promising way to design and fabricate highly efficient photocatalytic systems for overall water splitting.

Besides the development of metal-based semiconductors, several new types of photocatalysts have been also reported. Wang et al. [14] reported a visible light responsive metal-free polymeric photocatlayst of $\mathrm{C}_{3} \mathrm{~N}_{4}$, which showed activity for both water splitting half reactions under visible light. Liu et al. [15] found that some non-metal elements, such as $\mathrm{S}_{8}$ and $\mathrm{B}$, are also potential visible light active photo(electro)catalysts. Xu et al. [16] reported that a red metallic oxide photocatalyst $\left(\mathrm{Sr}_{1-x} \mathrm{NbO}_{3}\right)$ exhibited an absorption edge of $650 \mathrm{~nm}$ even without the introduction of nitrogen or sulfur.

Significant advances have been made in visible light driven photocatalytic water splitting for hydrogen production in this century, especially in the development of efficient visible light responsive semiconductors. This provides the basis for the design and assembly of highly efficient photocatalytic water splitting systems. It should be noted that although the theoretical requirement of the band gap for the photocatalytic one-step water splitting reaction is $1.23 \mathrm{eV}$, the actual band gap of the photocatalyst should be much larger than $1.8 \mathrm{eV}$ because of the existence of band bending and surface overpotential [7]. Fig. 2 shows the calculated solar energy conversion efficiency as a function of wavelength for overall water splitting using photocatalysts with various quantum efficiencies [8]. It was proposed that the solar energy conversion efficiency should reach at least $10 \%$ for commercial application. Even when a photocatalyst with absorption edge of ca. $500 \mathrm{~nm}$ achieves a quantum efficiency of $100 \%$, as shown in Fig. 2 , the solar energy conversion efficiency is less than $10 \%$. Comparatively, the target on the material with a $600 \mathrm{~nm}$ absorption edge will be achieved once its quantum efficiency is over $60 \%$. However, it



Fig. 2. Calculated solar energy conversion efficiency as a function of wavelength for overall water splitting using photocatalysts with various quantum efficiency. Solar irradiance used for the calculation was taken from AM 1.5G data. Reproduced from Ref. [8] by permission of The American Chemical Society. still remains challenging to achieve overall water splitting with $600 \mathrm{~nm}$ class materials. Photocatalytic water splitting for hydrogen production is a multidisciplinary topic, and it involves basic scientific principles from fields such as biological photosynthesis, photovoltaic cells and photoelectrocatalysis, etc. It is also of vital importance to understand the photocatalytic processes with the assistance of advanced techniques, such as theoretical calculation, ultrafast spectroscopy and in situ characterizations. Novel strategies and concepts similar to the cocatalyst and "junction" [17] should be developed to aid the design and fabrication of highly efficient photocatalytic systems. In summary, research on visible light driven photocatalytic water splitting is a long standing challenge which needs scientists to persevere with patience and courage and to strive without relaxation.

Fuxiang Zhang

Dalian Institute of Chemical Physics,

Chinese Academy of Scieneces

*Corresponding author.

Tel: +86-411-84379698

E-mail: fxzhang@dicp.ac.cn

Received 25 June 2014

Published 20 September 2014

DOI: 10.1016/S1872-2067(14)60183-2

\section{References}

[1] Bard A J, Fox M A. Acc Chem Res, 1995, 28: 141

[2] Wen F Y, Yang J H, Zong X, Ma Y, Xu Q Ma B J, Li C. Prog Chem (温. 福宇, 杨金辉, 宗旭, 马艺, 徐倩, 马保军, 李灿. 化学进展), 2009, 21: 2285

[3] Asai R, Nemoto H, Jia Q X, Saito K, Iwase A, Kudo A. Chem Commun, 2014, 50: 2543

[4] Liu G, Yin L C, Wang J Q, Niu P, Zhen C, Xie Y P, Cheng H M. Energy Environ Sci, 2012, 5: 9603

[5] Liu G, Wang L Z, Sun C H, Yan X X, Wang X W, Chen Z G, Smith S C, Cheng H M, Lu G Q. Chem Mater, 2009, 21: 1266

[6] Chen S S, Yang J X, Ding C M, Li R G, Jin S Q, Wang D E, Han H X, Zhang F X, Li C.J Mater Chem A, 2013, 1: 5651

[7] Maeda K, Domen K.J Phys Chem C, 2007, 111: 7851

[8] Maeda K, Domen K. J Phys Chem Lett, 2010, 1: 2655

[9] Maeda K, Teramura K, Domen K. J Catal, 2008, 254: 198

[10] Ohno T, Bai L, Hisatomi T, Maeda K, Domen K. J Am Chem Soc, 2012, 134: 8254

[11] Maeda K, Higashi M, Lu D L, Abe R, Domen K. J Am Chem Soc, 2010, 132: 5858

[12] Miseki Y, Fujiyoshi S, Gunji T, Sayama K. Catal Sci Technol, 2013, 3: 1750

[13] Sasaki Y, Nemoto H, Saito K, Kudo A. J Phys Chem C, 2009, 113: 17536

[14] Wang X C, Maeda K, Thomas A, Takanabe K, Xin G, Carlsson J M, Domen K, Antonietti M. Nat Mater, 2009, 8: 76

[15] Liu G, Niu P, Yin L C, Cheng H M. J Am Chem Soc, 2012, 134: 9070

[16] Xu X X, Randorn C, Efstathiou P, Irvine J T S. Nat Mater, 2012, 11: 595

[17] Yang J H, Wang D E, Han H X, Li C. Acc Chem Res, 2013, 46: 1900 